\title{
Perímetro de cuello y éxito del tratamiento de pacientes con obesidad: estudio de vida real
}

\author{
Martha Kaufer-Horwitz, * María Gisela Carreto-Adán y Fernando Pérez-Hernández
}

Instituto Nacional de Ciencias Médicas y Nutrición Salvador Zubirán, Clínica de Obesidad y Trastornos de la Conducta Alimentaria, Departamento de Endocrinología y Metabolismo, Ciudad de México, México

\section{Resumen}

Introducción: La evaluación del paciente con obesidad es un reto debido a las dificultades técnicas para efectuar las mediciones. Objetivo: Evaluar la asociación entre el perímetro de cuello (PCu) y el de cintura (PC) con marcadores de riesgo cardiometabólico y el éxito del tratamiento de pacientes con obesidad mórbida. Método: Se estudiaron 470 pacientes de $39.3 \pm 11.4$ años e índice de masa corporal de $44.1 \pm 8.4 ; 73.5 \%$ era del sexo femenino. Se evaluó índice de masa corporal, $P C, P C$, perímetro de cadera y marcadores cardiometabólicos basales y finales. Se definió como éxito a una pérdida ponderal $\geq 5 \%$. Resultados: Se encontraron correlaciones significativas entre $P C$ y $P C u$ y entre estos y marcadores de riesgo cardiometabólico, así como entre los cambios en PC y PCu y el éxito en el tratamiento. El PCu predijo el éxit̄̆ en modelos de regresión logística. Conclusiones: Se documentó la asociación entre PC y PCu con indicadores de riesgocardiometabólico y la asociación del PCu con éxito en el tratamiento en pacientes con obesidad mórbida. Dada la sencilleż de su obtención, el PCu podría sustituir al PC en la evaluación y seguimiento de pacientes con obesidad clase III.

PALABRAS CLAVE: Perímetro de cuello. Perímetro de cintura. Índice de masa corporal. Obesidad. Adultos.

\section{Abstract}

Introduction: Evaluation of the patient with obesity is a challenge due to the technical difficulties to carry out measurements. Objective: To assess the association between neck circumference (NC) and waist circumference (WC) with cardio-metabolic risk markers, as well as treatment success in patients with morbid obesity. Method: Four-hundred and seventy patients of 39.3 \pm 11.4 years of age and with a body mass index (BMI) of $44.1 \pm 8.4$ were studied; $73.5 \%$ were females. Baseline andifinal BMI, WC, NC, hip circumference and cardio-metabolic markers were assessed. Success was defined as weight loss $\geq ₫ 5 \%$. Results: Significant correlations were found between WC and NC, and between these and cardio-metabolic risk markers, as well as between changes in WC and NC and treatment success. NC predicted success in logistic regression models. Conclusions: The association of WC and NC with cardio-metabolic risk indicators and the association of NC with treatment success in patients with morbid obesity was documented. Given the simplicity for obtaining it, NC might replace WC in the assessment and follow-up of patients with class III obesity.

KEY WORDS: Neck circumference. Waist circumference. Body mass index. Obesity. Adults.

Correspondencia:

*Martha Kaufer-Horwitz

E-mail: marthakaufer@gmail.com
Fecha de recepción: 10-07-2019

Fecha de aceptación: 05-09-2019

DOI: $10.24875 / G M M .19005387$
Gac Med Mex. 2019;155:596-601 Disponible en PübMed www.gacetamedicademexico.com 


\section{Introducción}

La obesidad es un problema de salud pública a escala global. ${ }^{1}$ En México, la prevalencia de sobrepeso y de obesidad ha mostrado una tendencia ascendente desde 1988 y en 2016 alcanzó una prevalencia combinada (sobrepeso + obesidad) de $72.5 \%$ en adultos. ${ }^{2}$

La obesidad es una enfermedad caracterizada por aumento anormal de la adiposidad. Aun cuando el índice de masa corporal (IMC) mide masa y no adiposidad, se ha usado para definir y evaluar obesidad con fines epidemiológicos y clínicos. La medición de la grasa, particularmente visceral, requiere métodos poco accesibles en la práctica clínica, ${ }^{3}$ aunque existen alternativas como la medición de los perímetros de cintura (PC), cadera y, más recientemente, cuello $(\mathrm{PCu}){ }^{4}$ Se ha propuesto al IMC combinado con indicadores de adiposidad central como el PC, por ser predictivo de riesgo cardiometabólico. ${ }^{5}$ Sin embargo, aunque el PC es una medida práctica de bajo costo y su asociación con marcadores de riesgo metabólico es alta, existen problemas técnicos en personas con obesidad y se ha cuestionado su conveniencia en individuos con IMC $\geq 35$ por su baja precisión y reproducibilidad, la dificultad para identificar los referentes óseos ${ }^{6,7}$ y a que la medición pueda variar por la distensión posprandial, la respiración y la ropa. ${ }^{8,9}$

Al igual que el PC, el PCu es un indicador de adiposidad en la parte superior del cuerpo y de obesidad central, ${ }^{10}$ aunque su uso está menos generalizado y se carece de criterios unificados de riesgo. Es una medición de bajo costo, rápida y fácil de realizar por personal entrenado, más reproducible que el PC y se ha propuesto como alternativa para evaluar pacientes con sobrepeso u obesidad debido a que tiene menos dificultades técnicas, es más estable y no está sujeta a distensión posprandial. ${ }^{9}$

Se ha explorado la relación del PCu con el diagnóstico, evaluación y seguimiento de pacientes con sobrepeso u obesidad, ${ }^{9}$ síndrome de apnea-hipopnea obstructiva del sueño, ${ }^{11-13}$ resistencia a la insulina, ${ }^{14}$ hipertensión arterial ${ }^{15}$ o riesgo cardiovascular; ${ }^{16}$ además, se asocia con síndrome metabólico. ${ }^{6,17}$ Hasta donde conocemos, son pocos los estudios ${ }^{18,19}$ que exploran la relación entre el PC y el PCu en pacientes con obesidad mórbida, así como con el éxito en programas de tratamiento de obesidad.

En la Clínica de Obesidad y Trastornos de la Conducta Alimentaria del Instituto Nacional de Ciencias Médicas y Nutrición Salvador Zubirán se lleva a cabo el Programa de Atención al Paciente con Obesidad que ofrece tratamiento integral, con atención médica, hutriológica y psicológica, a partir de un programa psicoeducativo de 20 semanas con visitas mensuales. Incluye un plan de alimentación estandarizado, tratamientomédico de las comorbilidades y apoyo psicológico para promover el cumplimiento del tratamiento. Más de $70 \%$ de los pacientes cursa con obesidad mórbida (IMC $₫ 40$ $0 \geq 35$ en presencia de comorbilidades).

Con la finalidad de explorar la utilidad del PCu en cientes con obesidad, particularmente mórbida, el objetivo del presente estudio fue evaluar la asociación entre el PC y el PCu en pacientes que asisten a dicho programa y su asociación con marcadores de riesgo cardiometabólico, así como con el éxito en el tratamiento.

\section{Método}

Estudio de vida rea ${ }^{20}$ que consiste en un análisis retrospectivo de una cohorte de 470 pacientes de primera vez que ingresaron al Programa de Atención al Paciênte con Obesidad entre abril de 2004 y julio de 2010, concluyeron el programa y tuvieron datos completos dęlas variables antropométricas iniciales y finales. Se inclüyeron pacientes entre 18 y 59 años, con IMC $\geq 30$ yescolaridad mínima de primaria completa.

Se evaluó peso, estatura, PC, cadera y PCu. mediciones fueron realizadas por nutriólogos estandarizados utilizando métodos aceptados en consensos internacionales. ${ }^{21}$ El peso se obtuvo mediante un equipo de biompedancia eléctrica TANITA con capacidad de $250 \mathrm{~kg}$, con ropa ligera y sin zapatos; la estatura con un estadímetro de pared con flexómetro. Los perímetros se midieron con una cinta métricẩ de fibra de vidrio (SECA 201). El PC se midió en el pünto medio entre la parte inferior de las costillas y las crestas ilíacas a nivel de la línea axilar media a la a ătura de la cicatriz umbilical y el perímetro de cadera èn el punto máximo del perímetro de los glúteos. El Ê BCu se midió justo debajo de la prominencia de la laringe, con la cabeza en el plano de Frankfort. ${ }^{15,21}$ Se calcularon IMC $\left(\mathrm{kg} / \mathrm{m}^{2}\right)$ e índice cintura/cadera. Se midiö la tensión arterial usando un esfigmomanómetro anereide de pared con brazalete de tamaño adecuado. $\frac{22}{\alpha}$ Se determinaron glucosa en ayuno y perfil de lípî́los (colesterol total, colesterol-LDL, colesterol-HDE y triglicéridos) en el Laboratorio Central del Instifuto Nacional de Ciencias Médicas y Nutrición Salvădor Zubirán. Se definió síndrome metabólico como la p̈resencia de tres o más componentes de los criterios armonizados de Alberti. ${ }^{23}$ Se consideró éxito del Æ̇tratamiento a una reducción ponderal $\geq 5 \%{ }^{6}$ 
Tabla 1. Características clínicas y metabólicas iniciales y finales, por sexo

\begin{tabular}{|c|c|c|c|c|c|c|c|c|c|c|}
\hline & \multicolumn{5}{|c|}{ Hombres $(n=123)$} & \multicolumn{4}{|c|}{ Mujeres ( $n=347$ ) } & 5 \\
\hline & \multicolumn{2}{|c|}{ Basal } & \multicolumn{2}{|c|}{ Final } & $\mathbf{p}^{*}$ & \multicolumn{2}{|c|}{ Basal } & \multicolumn{2}{|c|}{ Final } & 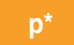 \\
\hline & \multicolumn{2}{|c|}{ Media \pm DE } & \multicolumn{2}{|c|}{ Media \pm DE } & & \multicolumn{2}{|c|}{ Media \pm DE } & \multicolumn{2}{|c|}{ Media \pm DE } & $\bar{\sigma}$ \\
\hline Edad (años) & \multicolumn{2}{|c|}{$38.6 \pm 11.9$} & & & & \multicolumn{2}{|c|}{$39.2 \pm 11.3$} & & & $\overline{2}$ \\
\hline Estatura (m) & \multicolumn{2}{|c|}{$1.71 \pm 0.07$} & & & & \multicolumn{2}{|c|}{$1.58 \pm 0.07$} & & & \\
\hline Peso (kg) & \multicolumn{2}{|c|}{$133.8 \pm 30.0$} & \multicolumn{2}{|c|}{$126.2 \pm 28.8$} & 0.000 & \multicolumn{2}{|c|}{$109.2 \pm 21.9$} & \multicolumn{2}{|c|}{$104.1 \pm 21.3$} & 0.000 \\
\hline IMC & \multicolumn{2}{|c|}{$45.4 \pm 8.9$} & \multicolumn{2}{|c|}{$42.8 \pm 9.0$} & 0.000 & \multicolumn{2}{|c|}{$43.7 \pm 8.2$} & \multicolumn{2}{|c|}{$41.7 \pm 8.0$} & 0.000 \\
\hline Perímetro de cintura (cm) & \multicolumn{2}{|c|}{$136.5 \pm 18.1$} & \multicolumn{2}{|c|}{$129.9 \pm 18.5$} & 0.000 & \multicolumn{2}{|c|}{$118.4 \pm 16.9$} & \multicolumn{2}{|c|}{$113.9 \pm 16.2$} & 0.000 \\
\hline Perímetro de cadera $(\mathrm{cm})$ & \multicolumn{2}{|c|}{$134.5 \pm 18.3$} & \multicolumn{2}{|c|}{$129.9 \pm 18.2$} & 0.000 & \multicolumn{2}{|c|}{$135.7 \pm 15.8$} & \multicolumn{2}{|c|}{$132.0 \pm 16.2$} & 0.000 \\
\hline Índice cintura/cadera & \multicolumn{2}{|c|}{$1.02 \pm 0.07$} & & .07 & 0.004 & 0.8 & 0.08 & & 0.07 & 0.000 \\
\hline Perímetro de cuello (cm) & & 3.4 & & 4.1 & 0.000 & & $=3.5$ & & 3.7 & 0.000 \\
\hline Tensión arterial sistólica (mm Hg) & 133 & 14.9 & & 13.5 & 0.000 & 129 & 15.2 & 125 & 14.1 & 0.000 \\
\hline Tensión arterial diastólica (mm Hg) & 87 & 10.8 & & 10.2 & 0.001 & 84. & 11.0 & & 9.3 & 0.000 \\
\hline Glucosa (mg/dL) & 101 & 20.1 & & 36.5 & 0.497 & 103 & 26.1 & & 21.6 & 0.000 \\
\hline Colesterol total (mg/dL) & 184 & 34.2 & & 38.4 & 0.036 & 190 & $=38.5$ & 184 & 37.3 & 0.006 \\
\hline Triglicéridos (mg/dL) & 182 & 78.0 & & 70.2 & 0.022 & 170 & 89.4 & 158 & 76.3 & 0.001 \\
\hline Colesterol-LDL (mg/dL) & 112 & 28.7 & & 37.4 & 0.035 & 118 & $=32.2$ & 108 & 40.8 & 0.001 \\
\hline Colesterol-HDL (mg/dL) & & 6.7 & & 8.2 & 0.301 & & 9.0 & & 9.1 & 0.752 \\
\hline Componentes síndrome metabólico\# & & 1.04 & & 1.05 & 0.001 & 3.4 & 1.03 & & 0.67 & 0.000 \\
\hline & n & $\%$ & n & $\%$ & $p^{\S}$ & $\mathrm{n}$ & $\%$ & $\mathrm{n}$ & $\%$ & p8ㅇㄴ \\
\hline Glucosa alterada & 50 & 40.7 & 26 & 21.1 & 0.000 & 148 & 42.7 & 65 & 18.7 & 0.000 \\
\hline Triglicéridos alterados & 68 & 55.5 & 44 & 35.8 & 0.001 & 168 & 48.4 & 100 & 28.8 & 0.000 \\
\hline Colesterol-HDL alterado & 97 & 78.9 & 62 & 50.4 & 0.000 & 296 & 85.3 & 203 & 58.5 & 0.000 \\
\hline Tensión arterial alterada & 95 & 77.2 & 64 & 52.0 & 0.000 & 211 & 60.8 & 160 & 46.1 & 0.041 \\
\hline Síndrome metabólico\# & 65 & 81.3 & 58 & 72.5 & 0.005 & 171 & 81.0 & 145 & 68.7 & $0 . \overline{000}$ \\
\hline
\end{tabular}

El estudio fue aprobado por los Comités de Ética en Investigación y de Investigación del propio Instituto. Los participantes otorgaron consentimiento informado por escrito al ingresar al Programa de Atención al Paciente con Obesidad.

Se determinaron medidas de tendencia central y de dispersión (media, desviación estándar) en las variables continuas y frecuencias y porcentajes en las categóricas. Se realizó un análisis de correlación simple ( $r$ de Pearson) entre las variables antropométricas y las de riesgo cardiometabólico y de Spearman entre éxito y los cambios en PC y PCu. Para evaluar las diferencias de medias (iniciales y finales) se utilizó $t$ pareada, asumiendo igualdad o no igualdad de las varianzas según los resultados de la prueba de
Levene $(F)$ o de chi cuadrada para variables catègóricas. Se generaron modelos de regresión logística para éxito en el tratamiento y se estimaron razones de momios y sus intervalos de confianza de $95 \%$. Todos los análisis se hicieron divididos por sexo y se consideró significación estadística con $p<0.05$. Se úifizó el programa SPSS versión 22.0 para Windows.

\section{Resultados}

Se estudiaron 470 pacientes con obesidad (IMC de 30.2 a 77.7$) ; 73.5 \%$ era del sexo femenino. La edad fue de $39.3 \pm 11.4$ años y el peso e IMC iniciales fueron $115.7 \pm 26.6 \mathrm{~kg}$ y $44.1 \pm 8.4$, respectivamente; $63.6 \%$ de los pacientes tenía un IMC $\geq 40$ (sin diferencias 
entre sexos), lo que indicaba obesidad clase III de acuerdo con los criterios de la Organización Mundial de la Salud. ${ }^{24}$ En ambos sexos, el PC indicó obesidad abdominal ${ }^{23}$ y el PCu presentó valores superiores a lo informado en distintos estudios. ${ }^{8}$ Los parámetros metabólicos tuvieron valores compatibles con los componentes del síndrome metabólico y solo el colesterol total mostró medias dentro de valores normales. Al inicio, todos los pacientes presentaron PC alterado y $78.9 \%$ de los hombres y $85.3 \%$ de las mujeres cursaron con hipoalfalipoproteinemia (Tabla 1).

Se encontraron diferencias significativas en PC, perímetro de cadera y PCu $(p<0.001)$ cuando se dividió a los pacientes por grupo de IMC $<35 \mathrm{y} \geq 35$. Las mujeres con IMC $\geq 35$ presentaron valores mayores para tensión arterial sistólica, tensión arterial diastólica, glucosa, y menores para colesterol-LDL y colesterol-HDL $(p<0.01$ para tensión arterial sistólica y tensión arterial diastólica y $\mathrm{p}<0.05$ en los demás casos) que las de $\mathrm{IMC}<35$. No se encontraron diferencias en los parámetros de riesgo cardiometabólico en hombres por grupo de IMC.

Se documentó mejoría significativa en los indicadores antropométricos, clínicos y metabólicos al final del programa en ambos sexos, excepto en glucosa en mujeres y colesterol-HDL en hombres y mujeres (Tabla 1).

Cuando se contrastaron las variables por grupo de IMC se encontraron valores significativamente mayores de peso, IMC, PC, cadera, PCu, tensión arterial sistólica, tensión arterial diastólica, glucosa, colesterol-LDL y colesterol-HDL en mujeres con IMC $\geq 35$, mientras que en hombres solo se encontraron diferencias en las variables antropométricas.

Hubo disminución significativa en la prevalencia de marcadores alterados, salvo para el PC. La evolución del síndrome metabólico y sus componentes se estudió en 80 hombres y 211 mujeres con datos completos en estos marcadores. Al inicio, $81.3 \%$ de los hombres y $81 \%$ de las mujeres cursaron con síndrome metabólico. Al final del programa se documentó reducción en el número de componentes del síndrome metabólico ( $p=0.001$ en hombres y $p=0.000$ en mujeres) y su frecuencia se redujo ( $p=0.005$ en hombres y $p=0.000$ en mujeres) (Tabla 1). La frecuencia de síndrome metabólico en mujeres con IMC $\geq 35$ fue mayor que en aquellas con IMC $<35$ (82.4\% versus $60.0 \%, p=0.000)$; esto no sucedió en los varones ( $84.1 \%$ versus $63.6 \%, p=0.202$ ).

Se encontró correlación positiva y significativa entre PC y PCu en hombres y en mujeres, 0.353 y 0.558 , respectivamente $(p<0.01)$. En hombres se documentaron correlaciones inversas del IMC, PC y PCu con colesterol y colesterol-LDL, y positivas del IMC y el PC
Tabla 2. Correlación de pearson entre las variables antropométricas y los indicadores cardiometabólicos al inicio, por sexo

\begin{tabular}{|c|c|c|c|c|}
\hline & Variables de riesgo & IMC & Cintura & puêllo \\
\hline \multirow[t]{7}{*}{ Hombres } & Glucosa & -0.099 & -0.073 & -0.084 \\
\hline & Triglicéridos & -0.075 & -0.087 & -0.049 \\
\hline & Colesterol & $-0.248^{* *}$ & $-0.239^{* *}$ & $-0.816^{*}$ \\
\hline & Colesterol -HDL & -0.167 & -0.148 & -0.126 \\
\hline & Colesterol -LDL & $-0.233^{*}$ & $-0.215^{*}$ & -0.228 \\
\hline & Tensión arterial sistólica & $0.232^{* *}$ & $0.235^{\star *}$ & \\
\hline & Tensión arterial diastólica & $0.213^{*}$ & $0.188^{*}$ & 0.166 \\
\hline \multirow[t]{7}{*}{ Mujeres } & Glucosa & $0.142^{* *}$ & $0.149^{* *}$ & 0.105 \\
\hline & Triglicéridos & 0.069 & 0.005 & $0.138^{*}$ \\
\hline & Colesterol & -0.026 & 0.036 & 88 \\
\hline & Colesterol-HDL & $-0.109^{*}$ & $-0.130^{*}$ & -0.126 \\
\hline & Colesterol-LDL & -0.030 & 0.018 & 0.056 \\
\hline & Tensión arterial sistólica & $0.214^{\star *}$ & $0.140^{*}$ & $0 . \frac{162}{0}$ \\
\hline & Tensión arterial diastólica & $0.199^{* *}$ & $0.153^{\star *}$ & 0.121 \\
\hline \multicolumn{4}{|c|}{$\begin{array}{l}\text { Se utilizaron los valores basales. } \\
{ }^{*} p<0.05 ;{ }^{* *} p<0.01\end{array}$} & \\
\hline
\end{tabular}

con tensión arterial sistólica y tensión arterial diastólica. En mujeres, se encontraron correlaciones significativas de IMC, PC y PCu con tensión arterial sistólica y đensión arterial diastólica y colesterol-HDL, del IMC y con glucosa y del PCu con triglicéridos. El sentidō de las correlaciones fue el esperado salvo para colesterol y colesterol-LDL en hombres (Tabla 2).

La mediana de pérdida ponderal en hombres fuê de $7.6 \mathrm{~kg}(-28.8 \mathrm{a}+9.2 \mathrm{~kg})$ y de $4.4 \mathrm{~kg}(-28.8 \mathrm{a}+7.2 \mathrm{~kg})$ en mujeres; $56.1 \%$ de los hombres y $42.1 \%$ deslas mujeres tuvieron pérdidas ponderales $\geq 5 \%$, eon diferencias significativas entre sexos $(p=0.007)$

Se encontraron correlaciones significativas entre los cambios en PC y PCu y el porcentaje de peso perdido o el éxito del tratamiento, con excepción de los cambios en PCu y porcentaje de peso perdidōen las mujeres con IMC $<35$. Las correlaciones fuëron mayores en hombres. EI PC mostró correlaciōnes mayores que el PCu. Destacan las altas correlaciones del PC con porcentaje de peso perdido y éxito en hombres y mujeres con IMC $<35$ (Tabla 3).

El PCu entró a todos los modelos de regresión, con excepción del modelo 4, mientras que el PC no entró en ninguno. El modelo 3 tuvo $r^{2}$ más alta y explicó $21.7 \%$ de la varianza de la variable dependiente féxito) con tres variables: edad, perímetro de cuellio y glucosa en ayunas (Tabla 4). 
Tabla 3. Correlación entre los cambios en perímetros de cintura y de cuello y variables de resultado (\% de peso perdido o éxito)

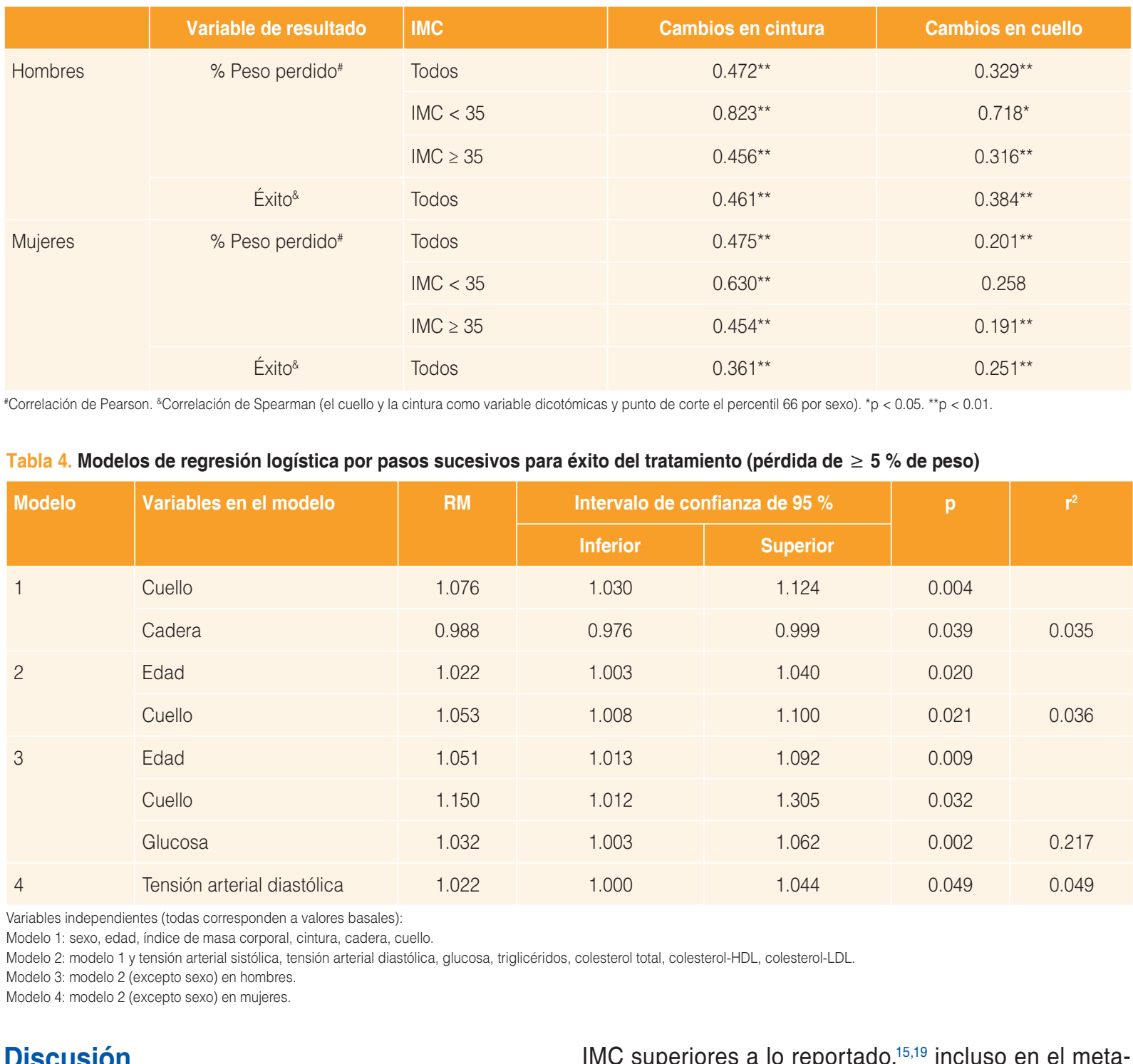

\section{Discusión}

El presente estudio evaluó la asociación entre PCu y PC con marcadores de riesgo cardiometabólico y el éxito del tratamiento en pacientes sometidos a un programa de tratamiento de la obesidad en una institución de tercer nivel de atención en el contexto de vida real. Hubo predominio de obesidad clase III y $45.7 \%$ de los pacientes tuvo éxito al alcanzar una pérdida ponderal $\geq 5 \%$ con mejoría en los indicadores de riesgo cardiometabólico.

Por la naturaleza de los pacientes, los valores de PCu fueron superiores a los puntos de corte reportados. ${ }^{8}$ Como en otros estudios, se identificó correlación significativa del PC con el PCu en hombres y mujeres, aunque con valores inferiores $(0.353$ en hombres y 0.558 en mujeres); sin embargo, la frecuencia de obesidad clase III fue alta y los valores de nálisis de Kroll et al. ${ }^{8}$

Aunque las correlaciones del PC y el PCu conolos marcadores de riesgo metabólico no fueron altas, resultaron significativas (Tabla 2), sin embargo se documentaron correlaciones mayores de los cambios en PC o en PCu a lo largo del tratamiento con variables de desenlace como porcentaje de peso perdido o éxito del tratamiento (Tabla 3) y superioridad relativa del PC sobre el PCu en todos los casos, incluso contrario a lo esperado en los pacientes con IMC $\geq 35$, donde la medición del PC suele ser imprecisa y poco reproducible. ${ }^{6,7}$ El PCu predijo el éxito en el tratamiento en los modelos de regresión logística, aunque con razones de momios y capacidad explicativa bajas.

Los resultados sugieren al PCu como indicador de adiposidad central dado que, como en otros estudios 5 -15-17 se asoció con indicadores de riesgo cardiovascular 
(colesterol total, colesterol-LDL, triglicéridos y tensión arterial). Se ha documentado que el PCu muestra una correlación alta $(r=0.4196, p<0.001)$, incluso mayor que el IMC, con riesgo de enfermedad coronaria a 10 años. ${ }^{9}$

Nuestro estudio tiene limitaciones: faltaron datos al ser un estudio de vida real, particularmente en las variables bioquímicas, posiblemente debido a la escasez de recursos de los pacientes o a la solicitud de estudios mínimos cuando los recursos son limitados. Sin embargo, los ensayos clínicos controlados no reflejan la práctica clínica habitual y se necesita generar evidencia de vida real para este fin. ${ }^{25}$ La prevalencia de síndrome metabólico puede estar subestimada en este estudio, al carecer de información de los pacientes con valores normales en las variables de riesgo que recibían tratamiento o que lo iniciaron durante el programa. El cambio en el PC debe tomarse con reserva en pacientes con IMC $\geq 35$. A pesar de esto, el estudio aporta información a la literatura existente al incluir una gran proporción de pacientes con obesidad clase III, con valores inusualmente altos de IMC (de 40 a 77), rara vez descritos, lo que permitió estudiar al PCu en una gama amplia de valores de IMC y su posible utilidad más allá de las medidas antropométricas clásicas. ${ }^{26}$

Si bien en el presente estudio el PCu no fue superior al PC, las correlaciones con éxito en el tratamiento y cambios metabólicos fueron similares y sugieren que el PCu podría ser una herramienta que ahorra tiempo en el consultorio y es menos invasiva que el PC.

En conclusión, el presente estudio documentó la asociación del PC y del PCu con indicadores de riesgo cardiometabólico y la asociación del PCu con el éxito en el tratamiento en pacientes con obesidad mórbida y podría ser una alternativa al PC en la evaluación, diagnóstico y seguimiento de estos pacientes. Al ser un indicador de adiposidad central y asociarse con comorbilidades frecuentes, el PCu podría complementar al IMC.

\section{Agradecimientos}

A los pacientes de la Clínica de Obesidad y Trastornos de la Conducta Alimentaria del Instituto Nacional de Ciencias Médicas y Nutrición Salvador Zubirán, así como al equipo multidisciplinario que les brinda atención de calidad.

\section{Bibliografía}

1. Independent Expert Group of the Global Nutrition Report. 2018 Global Nutrition Report: shining a light to spur action on nutrition. Reino Unido: Development Initiatives; 2018

2. Shamah-Levy T, Ruiz-Matus C, Rivera-Dommarco J, Kuri-Morales P, Cuevas-Nasu L, Jiménez-Corona ME, et al. Encuesta Nacional de Salud y Nutrición de Medio Camino 2016. Resultados Nacionales. Cuernavaca, México: Instituto Nacional de Salud Pública; 2017.

3. Lee SY, Gallagher, D. Assessment methods in human body composition. Curr Opin Clin Nutr Metab Care. 2008:11:566-572.

4. Hingorjo MR, Zehra S, Imran E, Qureshi MA. Neck circumferenge: a supplemental tool for the diagnosis of metabolic syndrome. J PakeMed Assoc. 2016;66:1221-1226.

5. Preis SR, Massaro JM, Hoffmann U, D'Agostino RB, Levy D, Robiñs SJ, et al. Neck circumference as a novel measure of cardiometabolic rišk: the Framingham Heart study. J Clin Endocrinol Metab. 2010;95:3701-3710.

6. Garvey WT, Mechanick JI, Brett EM, Garber AJ, Hurley DL, Jastreboff AM, et al. American Association of Clinical Endocrinologists and American College of Endocrinology comprehensive clinical practice guidelines for medicatcare of patients with obesity. Executive summary. Endocr Pract. 2016;22:842-884.

7. Klein S, Allison DB, Heymsfield SB, Kelley DE, Leibel RL, Nonas CTet al. Waist circumference and cardiometabolic risk: a consensus statement from Shaping America's Health: Association for Weight Management and Obesity Prevention; NAASO, the Obesity Society; the American Society for Nuffition; and the American Diabetes Association. Am J Clin Nutr. 2007;15:1061-4067.

8. Kroll C, Mastroeni SSBS, Czarnobay SA, Ekwaru JP, Veugeleß PJ, Mastroeni MF. The accuracy of neck circumference for assessing overweight and obesity: a systematic review and meta-analysis. Ann Hum Biol. 2017:44:667-677.

9. Koppad AK, Kaulgud RS, Arun BS. A study of correlation of neèk circumference with Framingham Risk Score as a predictor of coronany artery disease. J Clin Diagn Res. 2017:11:OC17-OC20.

10. Özkaya İ, Tunçkale A. Neck circumference positively related with central obesity and overweight in Turkish university students: a prelininary study. Cent Eur J Public Health. 2016;24:91-94.

11. Onat A, Hergenç G, Yüksel H, Can G, Ayhan E, Kaya Z, et al. NNeck circumference as a measure of central obesity: associations with metabolic syndrome and obstructive sleep apnea syndrome beyond waist circumference. Clin Nutr. 2009:28:46-51.

12. Tom C, Roy B, Vig R, Kang DW, Aysola RS, Woo MA, et al. Correlations between waist and neck circumferences and obstructive sleep axpnea characteristics. Sleep Vigil. 2018;2:111-118.

13. Martinho FL, Tangerina RP, Moura SM, Gregório LC, Túfik S, Bittencourt LR. Systematic head and neck physical examination as a predictor of obstructive sleep apnea in class III obese patients. Braz $\mathrm{J}$ Med Biol Res. 2008;41:1093-1097.

14. Da Silva CC, Zambon MP, Vasques AC, Rodrigues AM, Camilo DF. AAntonio MÂ, et al. Neck circumference as a new anthropometric indicator for prediction of insulin resistance and components of metabolic syndrome in adolescents: Brazilian Metabolic Syndrome Study. Rev Paul Pediatr. 2014;32:22'1-229.

15. Ben-Noun, L., Laor, A. Relationship between changes in neck circumference and changes in blood pressure. Am J Hypertens. 2004;17:409-414.

16. Borel AL, Coumes S, Reche F, Ruckly S, Pépin JL, Tamisier R, et al. Waist, neck circumferences, waist-to-hip ratio: Which is the best cardiometabolic risk marker in women with severe obesity? The SOON cohort. PLoS One. 2018;13:e0206617.

17. Luo $Y$, Ma $X$, Shen $Y, X u Y$, Xiong $Q$, Zhang $X$, et al. Neck circumference as an effective measure for identifying cardiometabolic syndrome: a comparison with waist circumference. Endocrine. 2017;55:822-830.

18. Assyov Y, Gateva A, Tsakova A, Kamenov Z. A comparison of the clinical usefulness of neck circumference and waist circumference in individuals with severe obesity. Endocr Res. 2017;42:6-14.

19. Yang L, Samarasinghe YP, Kane P, Amiel SA, Aylwin SJ. Viscerał, adiposity is closely correlated with neck circumference and represents a significant indicator of insulin resistance in WHO grade III obesity Clin Endocrinol (Oxf). 2010;73:197-200.

20. Ali MK, Echouffo-Tcheugui J, Williamson DF. How effective were lifestyle interventions in real-world settings that were modeled on the Diabletes Prevention Program? Health Aff (Millwood). 2012;31:67-75.

21. Lohman TG, Roche AF, Martorell R (editores.). Anthropometric standardization reference manual. EEUU: Human Kinetics Books; 1988. $\frac{\complement}{\alpha}$

22. Norma Oficial Mexicana NOM-030-SSA2-2009, para la prevención, detección, diagnóstico, tratamiento y control de la hipertensión anterial sistémica. México: Diario Oficial de la Federación; 2010.

23. Alberti KG, Eckel RH, Grundy SM, Zimmet PZ, Cleeman JI, Donato $\bar{K}$ A, et al. Harmonizing the metabolic syndrome: a joint interim statement of the International Diabetes Federation Task Force on Epidemiology and Prevention; National Heart, Lung, and Blood Institute; American Heart Association; World Heart Federation; International Atherosclerosis Society; and International Association for the Study of Obesity. Circulation. 2009;120:1640-9645.

24. Obesity: preventing and managing the global epidemic. Report of a $W H O$ Consultation (WHO Technical Report Series 894). Suiza: World Health Organization, 2000.

25. Neta G, Johnson KE Informing real-world practice with real-wor id evidence: the value of PRECIS-2. BMC Med. 2018;16:76.

26. Joshipura K, Muñoz-Torres F, Vergara J, Palacios C, Pérez CM. $\overline{\bar{N}}$ eckcircumference may be a better alternative to standard anthropometric measures. J Diabetes Res. 2016;2016:6058916. 